\title{
18F-FDG PETCT and 68Ga-DOTA PETCT Mismatch With in Vivo Histopathological Characterization of Low Grade Neuroendocrine Pancreatic Tumor
}

Marcello Moro Queiroz ( $\nabla$ mmqueiroz93@gmail.com )

Hospital Sirio-Libanes https://orcid.org/0000-0001-5789-3397

Carlos Diego Holanda Lopes

Hospital Sirio-Libanes

Alessandra Corte Real Salgues

Hospital Sirio-Libanes

Felipe de Galiza Barbosa

Hospital Sirio-Libanes

Emerson Shigueaki Abe

Hospital Sirio-Libanes

Thales Parenti Silveira

Hospital Sirio-Libanes

Marcel Cerqueira Cesar Machado

Hospital Sirio-Libanes

Fernanda Cunha Capareli

Hospital Sirio-Libanes

Case report

Keywords: neuroendocrine, low grade, 68Ga-DOTA PETCT, in vivo, mismatch, pancreatic tumor

Posted Date: January 15th, 2021

DOl: https://doi.org/10.21203/rs.3.rs-143517/v1

License: (c) (1) This work is licensed under a Creative Commons Attribution 4.0 International License.

Read Full License 


\section{Abstract}

\section{Background}

Pancreatic neuroendocrine tumor (PNET) is a subgroup of neuroendocrine tumor (NET) that has unique biology and natural history. The histological classification has a major role in the management of this pathology, but in recent years Gallium 68 dotatate (68Ga-DOTA) scanning is at the center of a discussion about how these imaging technologies can modify clinical management of neuroendocrine tumors and how their results are correlated to Ki67 index.

\section{Method}

We hereby describe a case of a patient that investigated an unspecific stable pancreatic nodule suspected of high-grade NET after evaluation with 68Ga-DOTATOC positron emission tomography computed tomography (PETCT) and ${ }^{18}$ F-Fluorodeoxyglucose $\left({ }^{18}\right.$ F-FDG) PETCT.

\section{Results}

The images corroborate the hypothesis of high-grade NET based on the standard uptake value (SUV) described in both image exams (16.4 in ${ }^{18}$ FDG PETCT and 9.2 in 68Ga-DOTATOC PETCT). After surgery, the histopathological analyses revealed a localized grade 2 well differentiated NET, Ki-67 of 4.7, evidencing a rare case of mismatch between the functional image and the in vivo characterization of the neoplasm.

\section{Conclusion}

Functional imaging of neuroendocrine tumors with different modalities of PETCT is a well-described strategy for evaluating PNET and can dictate conducts in some cases. However, histopathological analysis is crucial to confirm the grade and prognosis related to this disease. Besides the rarity of this case, our patient presented a mismatch between imaging and histopathological analysis, with the former one allowing active surveillance based on a low-grade neuroendocrine tumor.

\section{Introduction}

Neuroendocrine tumors (NET) are defined as epithelial neoplasms with predominant neuroendocrine differentiation and can arise in almost any organ of the body[1]. Pancreatic neuroendocrine tumors (PNET) are neoplasms that originate from the hormone-producing cells of the islets of Langerhans. They can be classified as functional or non-functional depending on whether they produce hormones that can cause symptoms and are relatively rare, accounting for approximately $1 \%$ of pancreatic cancers by incidence and $10 \%$ of pancreatic cancers by prevalence[2, 3$]$.

PNET also has distinct biological and clinical characteristics, like a high density of somatostatin receptors in well-differentiated cell membranes. Tumors defined as well-differentiated present a greater 
affinity for somatostatin, allowing the use of radiolabeled somatostatin analogs for imaging of these tumors[4-6]. Although Gallium 68 dotatate positron emission tomography - computed tomography (68 Ga-DOTA PETCT) is superior to ${ }^{18}$ F-Fluorodeoxyglucose $\left({ }^{18} \mathrm{FDG}\right)$ PETCT for imaging welldifferentiated NET, functional imaging with both 68 Ga-DOTA and ${ }^{18}$ FDG PETCT has the potential for a more comprehensive tumor assessment int intermediate and high-grade tumors[7].

We hereby report a case of a patient with PNET that was staged with 68 Ga-DOTA0 -Tyr3 octreotide (68 Ga-DOTATOC PETCT) and ${ }^{18}$ F-FDG PETCT. After this functional imaging assessment, the hypothesis of a high-grade neuroendocrine tumor was made, but the histopathological analysis confirmed a lowgrade NET, allowing active surveillance as a therapeutic option.

\section{Case Report}

A 48-year-old woman presented to the outpatient department with a history of an unspecific stable $1.0 \mathrm{~cm}$ hypervascular, solid nodule, localized in the uncinate process of the pancreas (Fig. 1) on two years of surveillance.

Because of irregular surveillance, an ${ }^{18} \mathrm{~F}-\mathrm{FDG}$ PETCT was performed and this nodule presented with high metabolic intake, standard uptake value (SUV)max of 16.4, apparently stable in size when compared to the previous exam (Fig. 2A). A complementary 68 Ga-DOTATOC PETCT was performed and revealed only the nodule in the uncinate process of the pancreas with an SUV max of 9.2 (Fig. 2B).

When compared to the previous magnetic resonance (MR) and FDG-PET with higher glycolytic metabolism, this set of information supported the diagnosis of poorly differentiated/high-grade neuroendocrine carcinoma. Additionally, the nodule was classified as non-functional based on a negative assessment of 5-hydroxy-indolacetic acid and chromogranin A. The patient was submitted to a pancreatic uncinectomy and the histopathologic sample evidenced a localized grade 2 well-differentiated neuroendocrine tumor of the pancreas with $0.9 \times 0.7 \mathrm{~cm}, \mathrm{Ki}-67$ of $4.7 \%$, pT1 (The American Joint Committee - AJCC 8th edition) (Fig. 3). Regarding the histopathological result and staging, we decided to maintain conservative management, with active surveillance and regular images of the abdomen. This unexpected functional image - histologic grade dissociation is rare and not yet described.

\section{Discussion}

We briefly describe a case of mismatch between in vivo histopathological characterization of PNET and imaging assessment by $68 \mathrm{Ga}$-DOTATOC PETCT and ${ }^{18}$ F-FDG PETCT. This report exemplifies how functional imaging can guide the management of NET, meanwhile highlight the importance of histopathological analysis in the treatment decision.

Peptides linked to DOTA and marked with $68 \mathrm{Ga}$, exemplified as DOTA0 -Tyr3 octreotate (DOTATATE), DOTATOC and DOTA0-1Nal3 octreotide (DOTANOC), bind specifically to somatostatin receptors (SSTR) in 
the cell surface membrane. Based on many previous studies, these modalities of PETCT are superior to many other image methods like computed tomography, MR and single photon emission computed tomography in the diagnoses of NET[8-10].

The incorporation of $68 \mathrm{Ga}$-labelled somatostatin analogs in PET imaging promoted a better diagnostic approach to NET, demonstrating high accuracy $(0,98$ in ROC analysis) combined with lower exam duration and radiation dose, in addition to better image resolution[11]. Early-stage lesions also benefit from this approach, as some of them are difficult to detect with conventional imaging, mostly because of their small size. However, as most of them are well-differentiated tumors, they present with higher expression of SSTR-2 and binding between the radiopeptide and the receptor[12].

Kayani et al exemplified the importance of using functional imaging with combined 68 Ga-DOTATATE and 18F-FDG PETCT in the assessment of neuroendocrine tumors. Based on a sample of 38 consecutive patients with the diagnosis of primary or recurrent NET, the combination of the two methods presented a sensitivity of $92 \%$, compared to $82 \%$ with 68 Ga-DOTATATE and $66 \%$ with 18 F-FDG PETCT alone.

Additionally, there was greater uptake of $68 \mathrm{Ga}$-DOTATATE than 18F-FDG in low grade NET (median SUV 29 vs $2.9, \mathrm{p}<.001$ ) and higher uptake of $18 \mathrm{~F}-\mathrm{FDG}$ over 68 Ga-DOTATATE in high grade NET (median SUV 11.7 vs $4.4, p=.03$ ). As a result, a significant correlation was achieved with predominant uptake of $68 \mathrm{Ga}-$ DOTATATE or $18 \mathrm{~F}-\mathrm{FDG}$ and tumor grade on histology $(\mathrm{p}<.0001)$, with the combination demonstrating the potential for a better comprehensive assessment in intermediate and high-grade tumors[7].

Several studies have suggested that patients with incidentally discovered, $<1 \mathrm{~cm}$ in size and low-grade tumors may be safely followed without surgery in some cases, depending on the site of the tumor[13, 14]. However, based on the possibility of high-grade tumors after functional imaging, we decided that surgery was the first treatment option and a complete histopathology analysis was possible. Early-stage by the AJCC 8th edition (pT1), localized grade 2 and well-differentiated histopathological characterization supported the decision for active surveillance after surgery.

\section{Conclusion}

We reported a case of a patient with an unspecific stable pancreatic nodule suspected of high-grade neuroendocrine tumor based on functional imaging with 68Ga-DOTATOC PETCT and ${ }^{18} \mathrm{~F}$-FDG PETCT. After surgery, the histopathological analysis confirmed a low grade, well-differentiated PNET. Despite this rare case of mismatch between the functional image and the in vivo characterization of the neoplasm, different modalities of PETCT remain a well-described strategy for evaluating PNET and can dictate treatment options. Nevertheless, histopathological analysis remains crucial to guide the management of this uncommon disease, allowing active surveillance after surgery in this case.

\section{Abbreviations}

${ }^{18} \mathrm{~F}-\mathrm{FDG}-{ }^{18} \mathrm{~F}-\mathrm{Fluorodeoxyglucose}$ 
68Ga-DOTA - Gallium 68 dotatate

AJCC - The American Joint Committee

DOTANOC - DOTA0-1Nal3 octreotide

DOTATATE - DOTA0 -Tyr3 octreotate

DOTATOC - DOTAO -Tyr3 octreotide

MR - Magnetic resonance

NET - Neuroendocrine tumor

PNET - Pancreatic neuroendocrine tumor

SSTR - Somatostatin receptors

SUV - Standard uptake value

\section{Declarations}

\section{Funding}

This study was funded by Hospital Sírio-Libanês

\section{Conflicts of Interest}

The authors have no relevant financial or non-financial interests to disclosure

\section{Availability of data and material}

Data sharing not applicable to this article as no datasets were generated of analysed during the current study

\section{Ethical approval}

Informed consent was obtained from the patient for publication of this case report and accompanying images.

\section{Consent to Participate}

Informed consent was obtained from the patient included in this study

\section{Consent to publish}

Patients signed informed consent regarding publishing their data 
Acknowledgments

We would like to thank the patient for allowing us to share his case details.

\section{Declaration of interests}

The authors declare that they have no known competing financial interests or personal relationships that could have appeared to influence the work reported in this paper

\section{Authors contributions}

Marcello Moro Queiroz: writing original draft, online drafting, data collection

Carlos Diego Holanda Lopes: writing original draft, online drafting, data collection

Alessandra Corte Real Salgues: writing-reviewing, supervision, methodology

Felipe de Galiza Barbosa: writing-reviewing, figures development

Emerson Shigueaki Abe: writing-reviewing, supervision, methodology

Thales Parenti Silveira: writing-reviewing, figures development, immunohistochemical analysis

Marcel Cerqueira Cesar Machado: writing-reviewing, supervision, methodology

Fernanda Cunha Capareli: Conceptualization, writing-reviewing and supervision

\section{References}

1. Kaewput C, Suppiah S, Vinjamuri S. Correlation between standardized uptake value of ${ }^{68}$ Ga-DOTANOC positron emission tomography/computed tomography and pathological classification of neuroendocrine tumors. World J Nucl Med 2018;17:34. https://doi.org/10.4103/wjnm.WJNM_16_17.

2. Parbhu SK, Adler DG. Pancreatic neuroendocrine tumors: contemporary diagnosis and management. Hospital Practice 2016;44:109-19. https://doi.org/10.1080/21548331.2016.1210474.

3. Yao JC, Eisner MP, Leary C, Dagohoy C, Phan A, Rashid A, et al. Population-Based Study of Islet Cell Carcinoma. Ann Surg Oncol 2007;14:3492-500. https://doi.org/10.1245/s10434-007-9566-6.

4. Caplin ME, Buscombe JR, Hilson AJ, Jones AL, Watkinson AF, Burroughs AK. Carcinoid tumour. The Lancet 1998;352:799-805. https://doi.org/10.1016/S0140-6736(98)02286-7.

5. Breeman WAP, de Jong M, de Blois E, Bernard BF, Konijnenberg M, Krenning EP. Radiolabelling DOTApeptides with 68Ga. Eur J Nucl Med Mol Imaging 2005;32:478-85. https://doi.org/10.1007/s00259004-1702-y.

6. Ezziddin S, Logvinski T, Yong-Hing C, Ahmadzadehfar H, Fischer H-P, Palmedo H, et al. Factors predicting tracer uptake in somatostatin receptor and MIBG scintigraphy of metastatic 
gastroenteropancreatic neuroendocrine tumors. J Nucl Med 2006;47:223-33.

7. Kayani I, Bomanji JB, Groves A, Conway G, Gacinovic S, Win T, et al. Functional imaging of neuroendocrine tumors with combined PET/CT using68Ga-DOTATATE (DOTA-DPhe1,Tyr3-octreotate) and18F-FDG. Cancer 2008;112:2447-55. https://doi.org/10.1002/cncr.23469.

8. Gabriel M, Decristoforo C, Kendler D, Dobrozemsky G, Heute D, Uprimny C, et al. 68Ga-DOTA-Tyr3octreotide PET in neuroendocrine tumors: comparison with somatostatin receptor scintigraphy and CT. J Nucl Med 2007;48:508-18. https://doi.org/10.2967/jnumed.106.035667.

9. Buchmann I, Henze M, Engelbrecht S, Eisenhut M, Runz A, Schäfer M, et al. Comparison of 68GaDOTATOC PET and 111 In-DTPAOC (Octreoscan) SPECT in patients with neuroendocrine tumours. Eur J Nucl Med Mol Imaging 2007;34:1617-26. https://doi.org/10.1007/s00259-007-0450-1.

10. Kabasakal L, Demirci E, Ocak M, Decristoforo C, Araman A, Ozsoy Y, et al. Comparison of ${ }^{68} \mathrm{Ga}$ DOTATATE and ${ }^{68} \mathrm{Ga}$-DOTANOC PET/CT imaging in the same patient group with neuroendocrine tumours. Eur J Nucl Med Mol Imaging 2012;39:1271-7. https://doi.org/10.1007/s00259-012-2123-y.

11. Velikyan I. The diversity of (68)Ga-based imaging agents. Recent Results Cancer Res 2013;194:10131. https://doi.org/10.1007/978-3-642-27994-2_7.

12. Fani M, Del Pozzo L, Abiraj K, Mansi R, Tamma ML, Cescato R, et al. PET of somatostatin receptorpositive tumors using $64 \mathrm{Cu}$ - and $68 \mathrm{Ga}$-somatostatin antagonists: the chelate makes the difference. $\mathrm{J}$ Nucl Med 2011;52:1110-8. https://doi.org/10.2967/jnumed.111.087999.

13. Lee LC, Grant CS, Salomao DR, Fletcher JG, Takahashi N, Fidler JL, et al. Small, nonfunctioning, asymptomatic pancreatic neuroendocrine tumors (PNETs): Role for nonoperative management. Surgery 2012;152:965-74. https://doi.org/10.1016/j.surg.2012.08.038.

14. Strosberg JR, Cheema A, Kvols LK. Stage I nonfunctioning neuroendocrine tumors of the pancreas: Surgery or surveillance? JCO 2011;29:349-349. https://doi.org/10.1200/jco.2011.29.4_suppl.349.

\section{Figures}




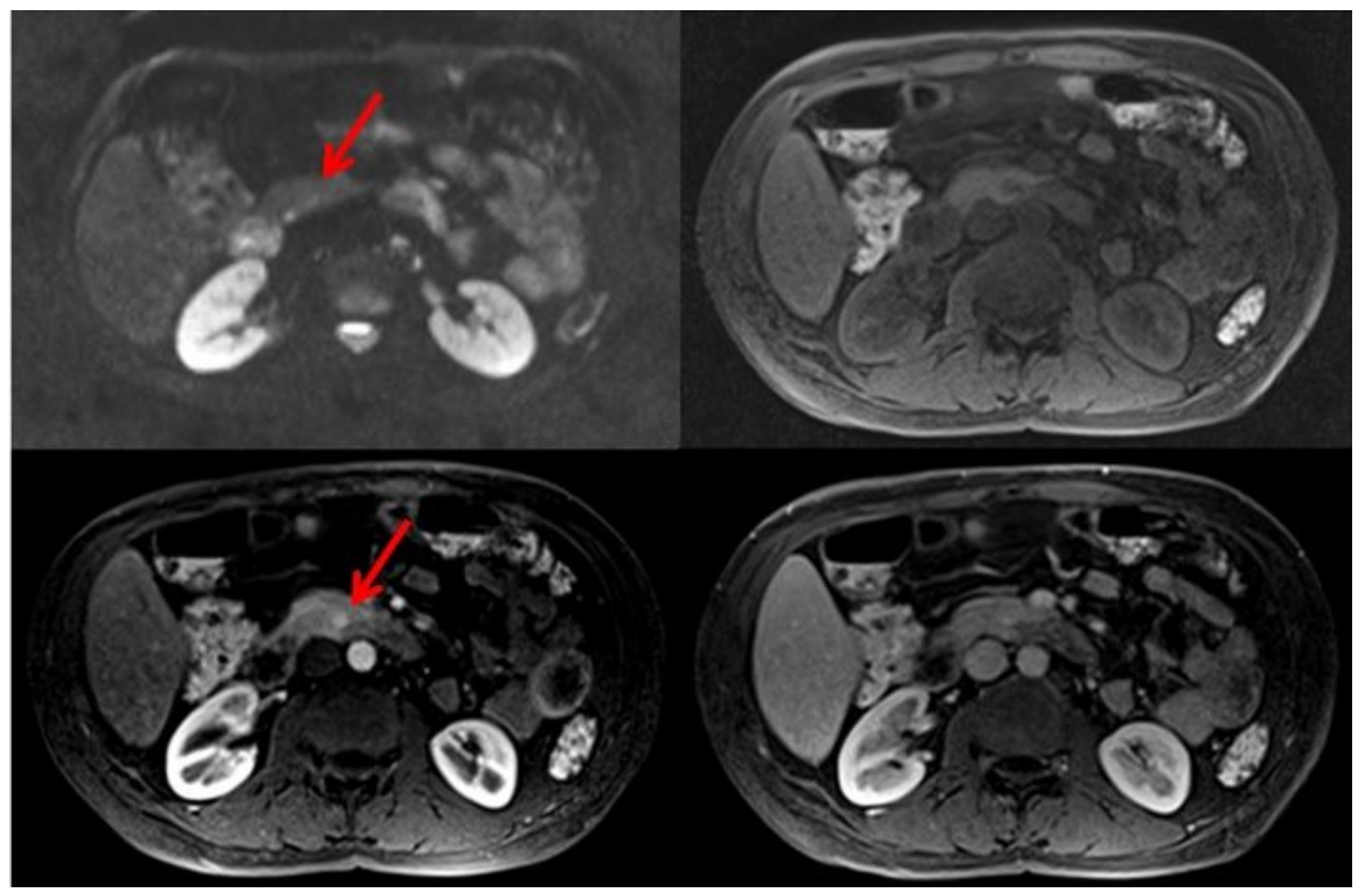

\section{Figure 1}

Magnetic Resonance images showing the hypervascular nodule in the pancreatic uncinate process (arrows). 
A

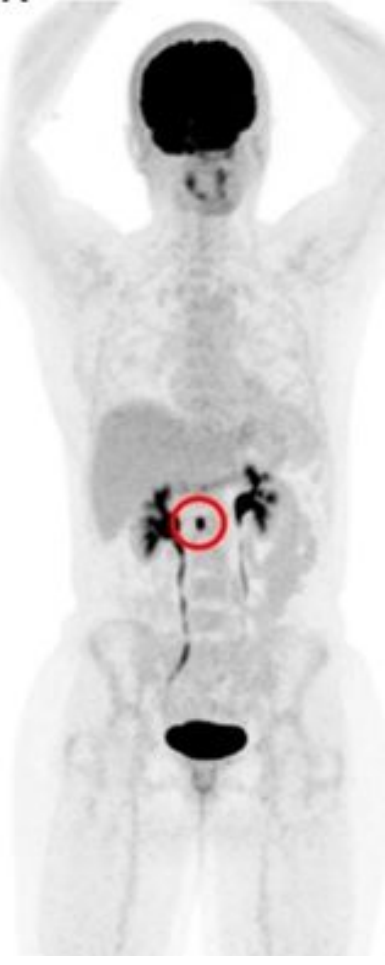

B
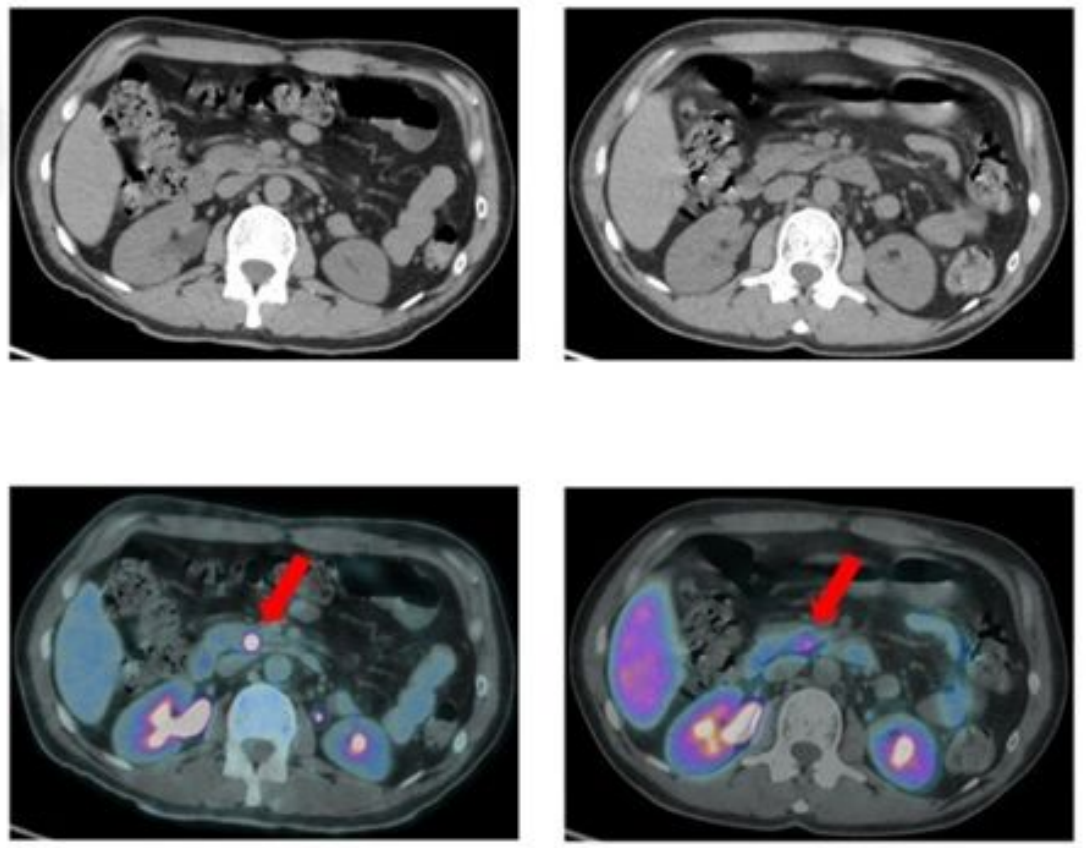

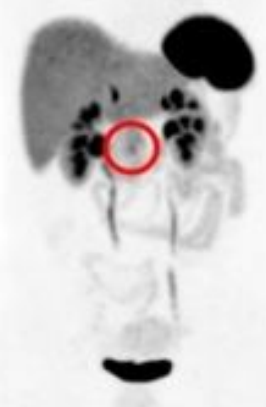

Figure 2

A: Initial 18F-FDG PET demonstrated a high metabolic expression in the uncinate pancreatic nodule (SUVmax: 16.4), apparently stable in size when compared to previous exams. B: 68Ga-DOTATOC shows the same nodule in the uncinate process of the pancreas with low somatostatin receptor expression (SUVmax 9.2), poorly characterized in the image without contrast,.
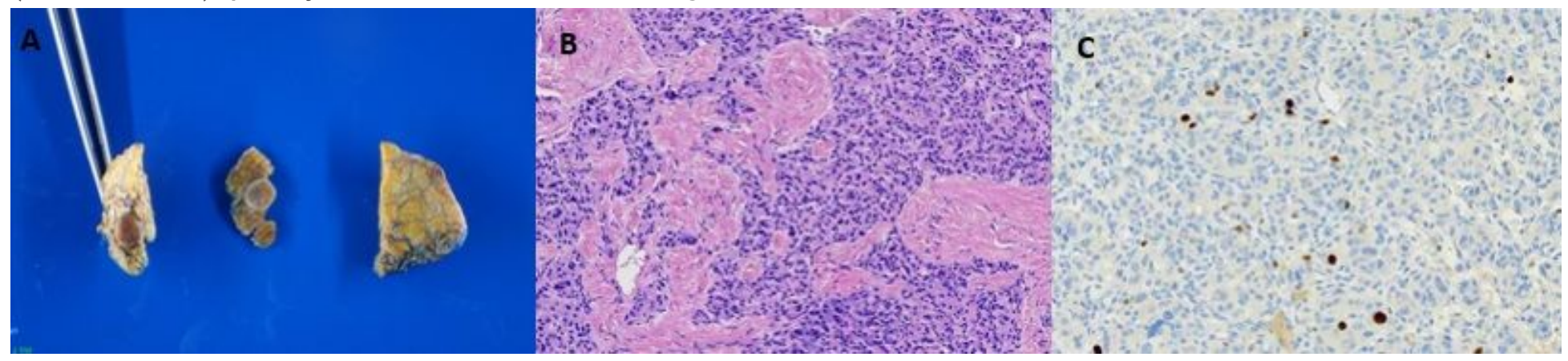

\section{Figure 3}

A: Macroscopic resected uncinate process of the pancreas, $3.7 \times 2,6 \times 0.6 \mathrm{~cm}$. In the center: Brown nodular lesion, well delimited, partial fibrosis involvement, $0.9 \times 0.7 \mathrm{~cm}, 0.3 \mathrm{~cm}$ distal from resection margin. B: unifocal pancreatic neoplasm with expansive growth. Cells with pleomorphic nuclei with eosinophilic cytoplasm. C: Proliferative index by Ki-67: 4.7\% (immunohistochemistry) 